\title{
BEMERKUNGEN ZUM SPEKTRALPROBLEM
}

\section{von D. Röddina und H. Schwichtenberg in Münster (Westfalen)}

Scholz stellte in [6] das Problem, ob sich für das System $\mathfrak{M}_{1}$ aller Spektren $S(\varphi):=\left\{m \mid e r f_{m+1} \varphi\right\}, \varphi$ Ausdruck der ersten Stufe (mit Identität, ohne Funktionssymbole), eine einfache zahlentheoretische Charakterisierung angeben läßt. Dieses Problem bleibt ungelöst; wir erhalten jedoch in Verschärfung von Resultaten von Asser [1] und Mostowski [4] eine Abschätzung $\operatorname{Pr}^{1}\left(\Im_{0}\right) \subseteq \operatorname{Ml}_{1} \subseteq \operatorname{Pr}^{1}\left(\Im_{1}\right)$ und allgemeiner $\operatorname{Pr}^{1}\left(\mathfrak{S}_{n}\right) \subseteq \mathfrak{M}_{n+1} \subseteq \operatorname{Pr}^{1}\left(\mathfrak{S}_{n+1}\right)$ mit $\mathfrak{M}_{n}:=\{\mathcal{S}(\varphi) \mid \varphi$ Ausdruck der $n$-ten Stufe $\}$ und Funktionenklassen $\mathfrak{\subseteq}_{n}$, die eine einigermaßen natürliche Klassifikation der elementaren Funktionen bilden (s. $\S 1)^{1}$ ). Insbesondere ergibt sich

1) $\mathfrak{M}_{1}$ enthält alle Mengen, deren charakteristische Funktion in GrzEGoRCzYK's (52 liegt.

2) $U_{n} \mathfrak{M}_{n}$ besteht aus allen Mengen mit elementarer charakteristischer Funktion.

3) $\mathfrak{M}_{n} \subset \mathfrak{M}_{n+2}$ (echte Inklusion).

Weiter betrachten wir die Systeme $\mathfrak{M}_{n+1}^{-}, n \geqq 1$, aller Spektren $S(\varphi)$ von Ausdrücken $\varphi$ der "schwachen $n+1$-ten Stufe" (freie Variable $n+1$-ter Stufe sind nicht zugelassen). Für $\mathfrak{M}_{n+1}^{-}$ergibt sich die folgende Charakterisierung: Ein (zahlentheoretisches) Prädikat heiße beschränkt exponentiell arithmetisch (b. e. a.), wenn es aus den Prädikaten $x+y=z, x \cdot y=z, 2^{x}=y$ mit Hilfe von Variablen für natürliche Zahlen, $\neg, \wedge, \wedge$ explizit definiert werden $\left.\mathbf{k a n n}^{2}\right)$. Dann besteht $\mathfrak{M}_{n+1}^{-}$aus allen Urbildern b. e. a. Mengen unter Funktionen der Form $\lambda m \vartheta_{n}\left((m+1)^{r}\right)$, $r \geqq 1$, mit $\vartheta_{0}(x):=x, \vartheta_{i+1}(x):=2^{\vartheta_{i}(x)}$.

\section{§ 1. Eine Klassifikation der elementaren Funktionen}

Die im folgenden verwendete Klassifikation der elementaren Funktionen wurde in [5] eingeführt und diskutiert. Wir stellen hier die später benötigten Definitionen und Sätze zusammen.

Eine (zahlentheoretische) Funktion heißt elementar, wenn sie explizit definiert werden kann mit Hilfe von Variablen für natürliche Zahlen, der Konstanten 1, den Funktionen,$+ \cdot$ und $[x / y]$ und den Operationen $\sum_{x<y}, \prod_{x<y}$; die Klasse der elementaren Funktionen bezeichnen wir mit $\&$. Einfache Abschlußeigenschaften

\footnotetext{
1) Mit $\operatorname{Pr}^{r}(\mathfrak{F})(\operatorname{Pr}(\mathfrak{F}))$ bezeichnen wir die Klasse aller $r$-stelligen (aller) Prädikate mit charakteristischer Funktion in $\mathfrak{F}$.

2) Nach Resultaten von BennetT (die uns aus einer Bemerkung in [8, p. 92] bekannt sind) stimmt die Klasse der b.e.a. Prädikate mit der Klasse der beschränkt arithmetischen Prädikate (ohne $2^{x}=y$ ) und mit der der rudimentären Prädikate im Sinne von Smullyan [8] überein.

1 ztschr. f. math. Logik
} 
elementarer Funktionen und Prädikate ${ }^{1}$ ) sind in [3, p. 285f.] zusammengestellt. Insbesondere ergibt sich, daß \& die kleinste Funktionenklasse ist, die die Funktion $x^{v}$, die Nachfolgerfunktion $N$, die konstanten Funktionen $C_{q}^{n}$ und die Identitätsfunktionen $U_{i}^{n}$ enthält und abgeschlossen ist gegen Einsetzungen und ,beschränkte primitive Rekursionen", d. h. solche primitive Rekursionen, bei denen die definierte Funktion durch eine schon vorher erzeugte Funktion beschränkt ist.

Wir nennen eine Funktion subelementar, wenn sie aus $\cdot N$, den $C_{q}^{n}$ und den $U_{i}^{n}$ mit Einsetzungen und beschränkten primitiven Rekursionen definierbar ist; die Klasse der subelementaren Funktionen bezeichnen wir mit $\mathfrak{S}$. $\mathcal{S}$ stimmt mit der von GRzEgorczYK in [2] eingeführten Funktionenklasse (52 überein. Zum Beispiel ist die Funktion $2^{x}$ nicht subelementar (aus Wachstumsgründen), wohl aber ihr Graph und auch die Funktion $\min \left(2^{x}, y\right)[5$, p. 207].

(E und 5 lassen sich auch durch Angabe von „Rechenzeit-Schranken" charakterisieren. Dazu beschreiben wir zunächst das Konzept einer Registermaschine (RM). Gegeben seien unendlich viele Register $R_{0}, R_{1}, R_{2}, \ldots$, von denen jedes eine natürliche Zahl $0,1,2, \ldots$ aufnehmen kann. Eine RM ist dann bestimmt durch ein Programm, d. h. eine endliche Liste von numerierten Instruktionen der folgenden Form:

$c A_{i} c^{\prime} \quad$ Addiere 1 zum Inhalt $a_{i}$ von $R_{i}$. Gehe über zur $c^{\prime}$-ten Instruktion.

$c S_{i} c^{\prime} \quad$ Subtrahiere 1 vom Inhalt $a_{i}$ von $R_{i}$, falls $a_{i} \neq 0$. Sonst lasse $a_{i}$ unverändert. Gehe über zur $c^{\prime}$-ten Instruktion.

$c P_{i} c_{0} c_{1}$ Prüfe den Inhalt $a_{i}$ von $R_{i}$. Ist $a_{i}=0(\neq 0)$, so gehe über zur $c_{0}\left(c_{1}\right)$-ten Instruktion.

$c$ Stop.

Bei vorgegebenen Anfangsinhalten $x_{0}, x_{1}, \ldots$ der Register beginnt die Rechnung einer RM mit der Ausführung der 0-ten Instruktion und setzt sich danach schritt. weise und determiniert fort, eventuell abbrechend. (Man beachte, daß jede RM nur mit endlich vielen Registern arbeitet, nämlich mit denen, auf die sich die Instruktionen beziehen. Die Inhalte der anderen Register haben auf die Rechnung keinen Einfluß, und sie werden im Verlauf der Rechnung nicht verändert.)

Eine $n$-stellige Funktion $f$ heißt RM-berechenbar, wenn es eine RM $M$ gibt, die, angesetzt auf die Registerinhalte $x_{0}, \ldots, x_{n-1}, 0,0, \ldots$, nach endlich vielen Schritten stoppt, und zwar mit den Registerinhalten $x_{0}, \ldots, x_{n-1}, f\left(x_{0}, \ldots, x_{n-1}\right), 0,0, \ldots$ Die Zahl der Rechenschritte von $M$ in Abhängigkeit von den Argumenten nennen wir eine Schriltzahlfunltion von $f$.

Durch Arithmetisieren des Rechenablaufs von RMn zeigt man wie üblich, daß genau die rekursiven Funktionen RM-berechenbar sind. Mit derselben Methode, wobei jedoch die auftretenden GöDEL-Numerierungen und Maschinenkonstruktionen geeignet festzulegen sind, ergibt sich: Eine Funktion ist subelementar (elementar)

1) Wie üblich nennen wir ein Prädikat elementar, wenn seine charakteristische Funktion elementar ist. Entsprechendes gilt auch für die im folgenden definierten Funktionenklassen. 
genau dann, wenn sie RM-berechenbar ist mit einer Schrittahlfunltion, die majorisierbar ist durch ein Polynom (in der Form $\vartheta_{n}\left(p(\mathfrak{x})\right.$ ), $p$ Polynom, $n \geqq 0$, wobei $\vartheta_{0}(x):=x$, $\left.\left.\vartheta_{i+1}(x):=2^{\vartheta_{i}(x)}\right)[5, \text { p. } 217 \mathrm{f} .]^{1}{ }^{1}\right)$

Es liegt jetzt nahe, die folgenden Teilklassen von $\&$ zu betrachten: $\mathfrak{S}_{n}(n \geqq 0)$ bestehe aus allen Funktionen $f$, die RM-berechenbar sind mit einer in der Form $\vartheta_{n}(p(\mathfrak{L})), p$ Polynom, majorisierbaren Schrittzahlfunktion. Offenbar ist $\mathfrak{S}_{0}=\mathfrak{S}$, $\mathfrak{S}_{n} \subseteq \mathfrak{S}_{n+1}$ und $\mathrm{U}_{n} \mathfrak{S}_{n}=\mathbb{E}$. Durch Konstruktion entsprechender RMn beweist man, $\operatorname{daB} \mathfrak{S}_{n}$ abgeschlossen ist gegen die Operationen $\underset{x<y}{\Sigma}, \underset{x<y}{\mu}$ und für $n \geqq 1$ gegen $\Pi$. $\operatorname{Pr}\left(\mathfrak{S}_{n}\right)$ ist abgeschlossen gegen $\neg, \wedge, \hat{\wedge}$. Ähnlich wie die obige Charakterisierung von $\mathbb{E}$ und $\mathfrak{S}$ ergibt sich, daß $\mathfrak{S}_{n}$ die kleinste Funktionenklasse $\mathfrak{F}$ ist mit (i) $\mathfrak{S} \subseteq \mathfrak{F}$, (ii) $\vartheta_{n} \in \mathfrak{F}$ und (iii) sind $g \in \mathfrak{S}$ und $h_{1}, \ldots, h_{r} \in \mathfrak{F}$, oder $g \in \mathfrak{F}$ und $h_{1}, \ldots, h_{r} \in \mathfrak{S}$, so liegt die durch $f(\mathfrak{x})=g\left(h_{1}(\mathfrak{x}), \ldots, h_{r}(\mathfrak{x})\right)$ definierte Funktion $f$ in $\mathfrak{F}\left[5\right.$, p. 218f.]. Die $\widetilde{S}_{n+1}$ sind jedoch nicht abgeschlossen gegen Einsetzungen (da $\vartheta_{n+1} \in \mathfrak{S}_{n+1}-\mathfrak{S}_{n}$ ) und auch nicht gegen beschränkte primitive Rekursionen [5, p. 219]. Ferner gibt es in $\mathfrak{S}_{n+1}$ eine Aufzählungsfunktion für die einstelligen Funktionen aus $\mathfrak{S}_{n}$, so daß also $\operatorname{Pr}\left(\mathfrak{S}_{n}\right) \subset \operatorname{Pr}\left(\mathfrak{S}_{n+1}\right)[5$, p. 219].

\section{Abschätzung der $\mathfrak{M}_{n}$ nach unten}

Wir zeigen, daß jede Menge $S$ mit charakteristischer Funktion in $\mathfrak{S}_{0}$ (also in GrzEgordzyK's (52) sich darstellen läßt als Spektrum eines Ausdrucks $\varphi$ der ersten Stufe (mit Identität, ohne Funktionssymbole) und allgemeiner, daß jede Menge $S$ mit charakteristischer Funktion in $\mathfrak{S}_{n}$ darstellbar ist als Spektrum eines Ausdrucks $\varphi$ der $n+1$-ten Stufe. Die Konstruktion von $\varphi$ orientiert sich an einer RM $M$, die die charakteristische Funktion von $S$ in $\leqq \vartheta_{n}(p(m))$ Schritten berechnet ( $p$ Polynom).

Wir beschreiben zunächst die zugrunde gelegte typentheoretische Sprache. Typenindizes oder kurz Typen seien $\iota$ und mit $\tau_{1}, \ldots, \tau_{r}$ auch $\left(\tau_{1}, \ldots, \tau_{r}\right)$. Für jeden Typ $\tau$ sollen abzählbar viele Variablen $x^{\tau}, y^{\tau}, \ldots$ zur Verfügung stehen. Ausdrücke sind $x^{\left(\tau_{1}, \ldots, \tau_{r}\right)} y_{1}^{\tau_{1}} \ldots y_{r}^{\tau_{r}}, x^{\tau}=y^{\tau}$ und mit $\varphi, \psi$ auch $\neg \varphi,(\varphi \wedge \psi), \underset{x^{\tau}}{\wedge} \varphi$. Wenn Mißverständnisse nicht zu befürchten sind, schreiben wir die Variablen auch ohne Typenindex. Die Stufe $|\tau|$ eines Typs $\tau$ erklären wir induktiv durch $|\iota|=0$, $\left|\left(\tau_{1}, \ldots, \tau_{r}\right)\right|=\max _{1 \leqq i \leqq r}\left(\left|\tau_{i}\right|+1\right)$. Die Stufe einer Variablen $x^{\tau}$ sei die Stufe von $\tau$. Ein Ausdruck $\varphi$ heißt von $n$-ter Stufe $(n \geqq 1)$, wenn alle in $\varphi$ gebundenen Variablen Stufen $\leqq n-1$ und alle in $\varphi$ freien Variablen Stufen $\leqq n$ haben. Wir schreiben $\operatorname{erf}_{m} \varphi$, wenn $\varphi$ eine erfüllende Interpretation über einem Bereich von $m$ Elementen besitzt; $S(\varphi):=\left\{m \mid e r f_{m+1} \varphi\right\}$ heißt Spektrum von $\varphi$. Das System aller Spektren von Ausdrücken $\varphi$ der $n$-ten Stufe bezeichnen wir mit $\mathfrak{M}_{n}$.

Satz 1. $\operatorname{Pr}^{1}\left(\mathfrak{S}_{n}\right) \subseteq \mathfrak{M}_{n+1}$.

Beweis. Sei $S \in \operatorname{Pr}^{1}\left(\Im_{n}\right)$. Wir konstruieren einen Ausdruck $\varphi$ der $n+1$-ten Stufe, so daß für $m \geqq 1$ gilt $m \in S \leftrightarrow e r f_{m+1} \varphi$. Dann ist $S$ im Fall $0 \in S$ das Spektrum von $\varphi \vee \underset{x}{\vee} \bigwedge_{y} x=y$, und im Fall $0 \notin S$ das Spektrum von $\varphi \wedge \underset{x}{\vee} \underset{y}{\vee} x \neq y$.

1) Mit $\mathfrak{x}, \mathfrak{y}, \mathfrak{z}, \ldots$ bezeichnen wir Variablentupel. 
Nach Definition von $\operatorname{Pr}^{1}\left(\mathfrak{S}_{n}\right)$ gibt es eine $\operatorname{RM} M$, die, angesetzt auf $m, 0, \ldots, 0$, in $\leqq \vartheta_{n}(p(m))$ Schritten stoppt ( $p$ Polynom), und zwar auf $m, 0, \ldots, 0$, falls $m \in S$, und auf $m, 1,0, \ldots, 0$, falls $m \notin S$. Der größte Index eines von $M$ bearbeiteten Registers sei $k$, und die Instruktionen von $M$ seien von 0 bis $l$ numeriert. Da in jedem Rechenschritt ein Registerinhalt höchstens um 1 vergrößert werden kann, lassen sich die im Verlauf der Rechnung auftretenden Registerinhalte abschätzen durch $m+\vartheta_{n}(p(m))$. Sei $r$ so gewählt, daß für $m \geqq 1$ gilt $m+\vartheta_{n}(p(m))<\vartheta_{n}\left((m+1)^{r}\right)$.

Wir geben zunächst $\varphi$ an. Sei $\sigma_{1}=(\iota \ldots \iota)$ mit $r$ Vorkommen von $\iota, \sigma_{i+1}=\left(\sigma_{i}\right)$. $x, y, z$ seien Variable des Typs $\iota, u, v, w$ Variable des Typs $\sigma_{n}$, falls $n \geqq 1$, und $r$-Tupel von Variablen des Typs $\iota$, falls $n=0$ (es ist klar, wie in diesem Fall etwa $u=v,{ }_{u}^{\wedge} \mathrm{zu}$ verstehen sind). Die Typen der restlichen Variablen (für die wir aus Gründen der Lesbarkeit große lateinische Buchstaben verwenden) ergeben sich aus dem Zusammenhang. $\varphi$ sei die Konjunktion der folgenden Ausdrücke.

$$
\begin{aligned}
& \bigwedge_{u} \neg K u u, \quad \bigwedge_{u} \bigwedge_{v}(K u v \vee K v u \vee u=v), \quad \bigwedge_{u} \bigwedge_{v} \underset{w}{\wedge}(K u v \wedge K v w \rightarrow K u w), \\
& \wedge_{u v}(N u v \leftrightarrow K u v \wedge \neg \underset{w}{\vee}(K u w \wedge K w v)) . \\
& \wedge_{x} \neg K^{\prime} x x, \quad \bigwedge_{x} \bigwedge_{y}\left(K^{\prime} x y \vee K^{\prime} y x \vee x=y\right), \wedge_{x}^{\wedge} \bigwedge_{z}\left(K^{\prime} x y \wedge K^{\prime} y z \rightarrow K^{\prime} x z\right), \\
& \wedge_{x} \underset{y}{\wedge}\left(N^{\prime} x y \leftrightarrow K^{\prime} x y \wedge \neg \underset{z}{\bigvee}\left(K^{\prime} x z \wedge K^{\prime} z y\right)\right) \\
& \hat{\wedge}_{x} \vee_{u} F x u, \quad \wedge_{x} \wedge \wedge_{v}(F x u \wedge F x v \rightarrow u=v), \quad \wedge_{x} \wedge_{y} \wedge_{u}(F x u \wedge F y u \rightarrow x=y), \\
& \bigwedge_{x} \bigwedge_{y} \bigwedge_{u} \bigwedge_{v}\left(F x u \wedge F y v \wedge N^{\prime} x y \rightarrow N u v\right), \quad \Lambda_{u}^{\wedge}\left(\neg \underset{v}{\vee} K v u \rightarrow{\underset{x}{\vee}}^{\vee} F x u\right) \\
& \wedge_{u} \vee R_{i} u v(i=0, \ldots, k), \quad \wedge_{u} \wedge_{v} \wedge_{w}\left(R_{i} u v \wedge R_{i} u w \rightarrow v=w\right)(i=0, \ldots, k), \\
& \hat{\Lambda}_{u} \underset{0 \leqq j \leqq l}{\vee} Z_{j} u, \quad \wedge_{u} \underset{0 \leqq i<j \leqq l}{\wedge} \neg\left(Z_{i} u \wedge Z_{j} u\right), \quad \bigwedge_{u}^{\wedge}\left(\neg \underset{v}{\vee} K v u \rightarrow Z_{0} u \wedge \hat{1 \leqq i \leqq k}_{i} R_{i} u u\right), \\
& \hat{y}_{y}^{\wedge} \wedge \wedge_{v}^{\wedge}\left(\neg \underset{x}{\vee} K y x \wedge \neg \underset{w}{\vee} K w u \wedge F y v \rightarrow R_{0} u v\right)
\end{aligned}
$$

Weiter gehört zu jeder Instruktion von $M$ ein Konjunktionsglied von $\varphi$, und zwar zu $c A_{i} c^{\prime}: \quad \wedge_{u} \bigwedge_{u^{\prime}}\left(Z_{c} u \wedge N u u^{\prime} \rightarrow Z_{c^{\prime}} u^{\prime} \wedge \bigwedge_{v} \bigwedge_{v^{\prime}}\left(R_{i} u v \wedge N v v^{\prime} \rightarrow R_{i} u^{\prime} v^{\prime}\right) \wedge\right.$

$$
\left.\wedge \underset{\substack{0 \leqq j \leq k \\ j \neq i}}{\wedge} \wedge_{v}^{\wedge}\left(R_{j} u v \rightarrow R_{j} u^{\prime} v\right)\right),
$$

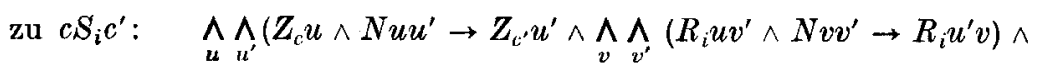

$$
\left.\wedge \wedge_{v}^{\wedge}\left(R_{i} u v \wedge \neg \underset{w}{\vee} K w v \rightarrow R_{i} u^{\prime} v\right) \wedge \underset{\substack{0 \leqq j \leqq k \\ j \neq i}}{\wedge} \wedge\left(R_{j} u v \rightarrow R_{j} u^{\prime} v\right)\right),
$$

zu $c P_{i} c_{0} c_{1}: \bigwedge_{u}^{\wedge} \bigwedge_{u^{\prime}}\left(Z_{c} u \wedge N u u^{\prime} \rightarrow\left(\underset{v}{\vee}\left(R_{i} u v \wedge \neg \underset{w}{\vee} K w v\right) \rightarrow Z_{c_{0}} u^{\prime}\right)\right.$

$$
\left.\left.\wedge\left(V_{v} R_{i} u v \wedge \underset{w}{\vee} K w v\right) \rightarrow Z_{c_{1}} u^{\prime}\right) \wedge \underset{0 \leqq j \leqq k}{\wedge} \underset{v}{\wedge}\left(R_{j} u v \rightarrow R_{j} u^{\prime} v\right)\right)
$$

zu $c$ Stop: $\wedge_{u} \bigwedge_{u^{\prime}}\left(Z_{c} u \wedge N u u^{\prime} \rightarrow Z_{c} u^{\prime} \wedge \hat{0 \leqq j \leqq k}_{v} \wedge\left(R_{j} u v \rightarrow R_{j} u^{\prime} v\right)\right)$

Das letzte Konjunktionsglied von $\varphi$ sei $\bigwedge_{u} \underset{v}{\wedge}\left(\neg \underset{w}{\bigvee} K u w \wedge \neg \underset{w}{\bigvee} K w v \rightarrow R_{1} u v\right)$. 
Wir zeigen jetzt $\operatorname{erf}_{m+1} \varphi \rightarrow m \in S$ für $m \geqq 1$. Gegeben sei also eine erfüllende Interpretation von $\varphi$ über einem $m+1$-elementigen Bereich. Man beachte zunächst, daß es genau $\vartheta_{n}\left((m+1)^{r}\right)$ Objekte des Typs $\sigma_{n}$ gibt; der Fall $n=0$ läßt sich einschließen, wenn man unter Objekten des Typs $\sigma_{0} r$-Tupel von Elementen des Bereichs versteht. Aus der Konstruktion von $\varphi$ ergibt sich: Das $K$ zugeordnete Objekt $\boldsymbol{K}$ ist eine lineare Ordnung der Objekte des Typs $\sigma_{n}$, und $\boldsymbol{N}$ ist die Nachfolgerrelation bzgl. $\boldsymbol{K}$. Entsprechend ist $\boldsymbol{K}^{\prime}$ eine lineare Ordnung der Elemente des Bereichs und $\boldsymbol{N}^{\prime}$ die Nachfolgerrelation bzgl. $\boldsymbol{K}^{\prime} . \boldsymbol{F}$ ist der Graph der ordnungstreuen (bzgl. $\left.\boldsymbol{K}^{\prime}, \boldsymbol{K}\right)$ Einbettung der Elemente des Bereichs auf das entsprechende Anfangsstück der Objekte des Typs $\sigma_{n} . \boldsymbol{R}_{i}$ ist der Graph einer Abbildung der Objekte des Typs $\sigma_{n}$ in sich, welche dem (bzgl. $\boldsymbol{K}$ ) $s$-ten Objekt des Typs $\sigma_{n}$ das $a$-te zuordnet, falls $M$, angesetzt auf $m, 0, \ldots, 0$, nach $s$ Schritten im $i$-ten Register die Zahl $a$ stehen hat, oder nach weniger als $s$ Schritten mit $a$ im $i$-ten Register stoppt. $Z_{j}$ trifft auf das (bzgl. $\boldsymbol{K}$ ) $s$-te Objekt des Typs $\sigma_{n}$ genau dann zu, wenn $M$, angesetzt auf $m, 0, \ldots, 0$, nach $s$ Schritten die $j$-te Instruktion ausführt, oder nach weniger als $s$ Schritten nach Ausführung der $j$-ten Instruktion stoppt. Aus dem letzten Konjunktionsglied folgt jetzt auf Grund der vorausgesetzten Eigenschaften von $M$ die Behauptung $m \in S$. Die Umkehrung $m \in S \rightarrow \operatorname{erf}_{m+1} \varphi$ für $m \geqq 1$ ergibt sich, indem man $K, K^{\prime}$ durch lineare Ordnungen des entsprechenden Typs und die restlichen freien Variablen durch die eben beschriebenen Objekte interpretiert. Damit ist der Satz bewiesen.

\section{§. Charakterisierungen der $\mathfrak{M}_{n}^{-}$, Abschätzung der $\mathfrak{M}_{n}$ nach oben}

Das Spektrum eines exponentiell arithmetischen (e. a.) abgeschlossenen Ausdrucks $\alpha$ sei die Menge aller $m$, so daß $\alpha$ in $\{x \mid x<m+1\}$ (im Standardsinn) gültig ist. Wir zeigen, daß sich das System $\mathfrak{M}_{n+1}^{-}$aller Spektren von Ausdrücken der schwachen $n+1$-ten Stufe (freie Variable $n+1$-ter Stufe sind nicht zugelassen) charakterisieren läßt als das System aller Urbilder von Spektren e. a. abgeschlossener Ausdrücke unter Funktionen der Form $\lambda m \vartheta_{n}\left((m+1)^{r}\right)-1, r \geqq 1$. Daraus ergibt sich als Korollar, daß $\mathfrak{M}_{n+1}^{-}$auch charakterisiert werden kann als das System aller Urbilder beschränkt e. a. Mengen unter Funktionen der Form $\lambda m \vartheta_{n}\left((m+1)^{r}\right)$, $r \geqq 1$. Weiter folgt mit $\S 1 \mathfrak{M}_{n+1}^{-} \subseteq \operatorname{Pr}^{1}\left(\Im_{n}\right)$, also erst recht $\mathfrak{M}_{n} \subseteq \operatorname{Pr}^{1}\left(\Im_{n}\right)$, und daraus dann $\mathfrak{M}_{n} \subset \mathfrak{M}_{n+2}$ und $\bigcup_{n} \mathfrak{M}_{n}=\operatorname{Pr}^{1}(\mathbb{E})$.

Wie bereits erwähnt, verstehen wir unter einem Ausdruck $\varphi$ der schwachen $n+1$-ten Stufe $(n \geqq 1)$ einen typentheoretischen Ausdruck (s. $\S 2$ ), in dem alle (gebundenen und freien) Variablen Stufen $\leqq n$ besitzen. Exponentiell arithmetische Ausdrücke seien $x+y=z, x \cdot y=z, 2^{x}=y$ (die mit $x, y, z$ bezeichneten Variablen sind nicht notwendig verschieden) und mit $\alpha, \beta$ auch $\neg \alpha,(\alpha \wedge \beta), \wedge_{x} \alpha$.

Satz 2. Zu jedem Ausdruck $\varphi$ der schwachen $n+$ 1-ten Stufe gibt es einen exponentiell arithmetischen abgeschlossenen Ausdruck $\alpha$, so daß mit einem geeigneten $r \geqq 1$ für alle $m \geqq 1$ gilt: erf $f_{m} \varphi$ genau dann, wenn $\alpha$ (im Standardsinn) güllig ist in $\left\{x \mid x<\vartheta_{n}\left(m^{r}\right)\right\}$. 
Beweis. Es genügt, dies für alle $m \geqq 2$ zu beweisen: Mit

$$
\delta: \equiv \bigvee_{x_{0}, \ldots, x_{\theta_{n}(1)} 0 \leqq i<j \leqq \theta_{n}(1)} x_{i} \neq x_{j}
$$

erfüllen dann $\neg \delta \vee \alpha$ im Fall $e r f_{1} \varphi$ und $\delta \wedge \alpha \operatorname{im~Fall~nicht~erf~} f_{1} \varphi$ die Behauptung.

Wir geben zunächst für jeden Typ $\tau$ eine GöDEL-Numerierung der Objekte des Typs $\tau$ über einem $m$-elementigen Bereich an. Dabei sei eine bijektive Zuordnung der Zahlen $0, \ldots, k(\iota)-1, k(\iota):=m$, zu den Objekten des Typs $\iota$ vorgegeben. Es sei nun $\tau=\left(\tau_{1}, \ldots, \tau_{r}\right)$, und die Objekte des Typs $\tau_{i}$ seien bereits mit den Zahlen $0, \ldots, k\left(\tau_{i}\right)-1$ numeriert. Es gibt dann $2^{k\left(\tau_{1}\right) \ldots k\left(\tau_{r}\right)}=: k(\tau)$ Objekte des Typs $\tau$. Wir definieren die GöDEL-Nummer von $x^{\tau}$ durch

mit

$$
g\left(x^{\tau}\right)=\sum_{i<k\left(x_{1}\right) \ldots k\left(r_{r}\right)} a_{i} \cdot 2^{i}
$$

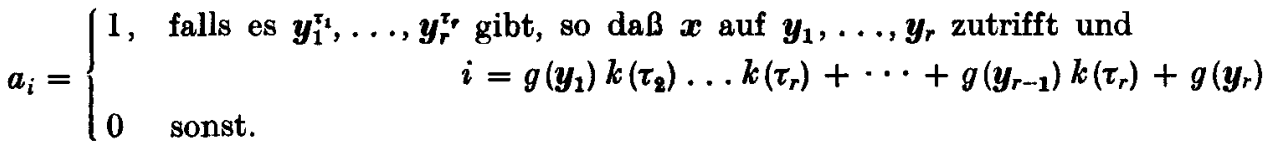

Offenbar trifft dann $x$ auf $y_{1}, \ldots, y_{r}$ genau dann zu, wenn gilt

wobei

$$
\neg \operatorname{Div}\left(\left[g(x) / 2^{g\left(y_{1}\right) k\left(x_{2}\right) \ldots k\left(x_{r}\right)+\cdots+g\left(y_{r-1}\right) k\left(z_{r}\right)+g\left(y_{r}\right)}\right], 2\right),
$$

$$
\operatorname{Div}(x, y) \leftrightarrow \underset{z}{\bigvee} x=y \cdot z
$$

Hier und im folgenden verwenden wir einige eingebürgerte zahlentheoretische Bezeichnungen zur Bildung arithmetischer Ausdrücke in einem weiteren Sinn; deren Gültigkeit bezieht sich dabei immer auf das Standardmodell über den natürlichen Zahlen.

Wir definieren jetzt zu jedem typentheoretischen Ausdruck $\psi$ einen arithmetischen Ausdruck (im weiteren Sinn) $\beta(\psi)$. Dabei legen wir eine eineindeutige Zuordnung von Variablen für natürliche Zahlen zu typentheoretischen Variablen zugrunde. Ist im folgenden eine typentheoretische Variable mit $x^{\tau}, y^{\tau}, y_{1}^{\tau}, \ldots$ bezeichnet, so bezeichnen wir die zugehörige zahlentheoretische Variable mit $x, y, y_{1}, \ldots k(\tau)$ steht für den entsprechend der obigen induktiven Definition gebildeten Term, welcher die Zahlkonstante $m$ enthält.

$$
\begin{gathered}
\beta\left(x^{\tau} y_{1}^{\tau_{1}} \cdots y_{r}^{\tau_{r}}\right) \equiv \neg \operatorname{Div}\left(\left[x / 2^{\left.y_{1} k\left(\tau_{s}\right) \ldots k\left(\tau_{r}\right)+\cdots+y_{r-1} k\left(\tau_{r}\right)+y_{r}\right]}, 2\right), \quad \beta\left(x^{\tau}=y^{\tau}\right) \equiv x=y,\right. \\
\left.\beta(\neg \psi) \equiv \neg \beta(\psi), \quad \beta\left(\psi_{1} \wedge \psi_{2}\right) \equiv \beta\left(\psi_{1}\right) \wedge \beta\left(\psi_{2}\right), \quad \beta \underset{x^{\tau}}{\wedge} \psi\right) \equiv{\underset{x<k(\tau)}{\wedge} \beta(\psi) .}^{\wedge} \beta(x)
\end{gathered}
$$

Es sei nun $\varphi$ ein Ausdruck der schwachen $n+1$-ten Stufe. Wir können annehmen, daß $\varphi$ abgeschlossen ist (sonst partikularisiere man vorn alle frei vorkommenden Variablen). Man zeigt dann leicht, daß erf $f_{m} \varphi$ genau dann, wenn $\beta(\varphi)$ gültig ist. 
Wir wollen jetzt $\beta(\varphi)$ äquivalent (bzgl. der Gültigkeit im Standardmodell) so umformen, daß schließlich ein pränexer abgeschlossener Ausdruck entsteht, dessen Kern aus Primformeln der Form $x+y=z, x \cdot y=z, 2^{x}=y$ gebildet ist (also die Zahlkonstante $m$ nicht enthält), und in dessen Präfix alle Quantoren durch $<\vartheta_{n}\left(m^{r}\right)$ mit festem $r \geqq 1$ beschränkt sind. Läßt man dann die Quantorenschranken weg, so entsteht der gesuchte exponentiell arithmetische Ausdruck $\alpha(\varphi)$.

Zur Umformung von $\beta(\varphi)$ verwenden wir zunächst die folgenden Äquivalenzen:

$$
\begin{aligned}
& \operatorname{Div}\left(\left[x / 2^{y}\right], 2\right) \leftrightarrow \underset{z \leqq x}{\vee}\left(z=\left[x / 2^{y}\right] \wedge \underset{u \leqq z}{\vee} u+u=z\right), \\
& z=\left[x / 2^{y}\right] \leftrightarrow\left(\neg \underset{u \leqq x}{\vee} u=2^{y} \wedge z=0\right) \vee \underset{u \leqq x}{\vee}\left(u=2^{y} \wedge \underset{v<u}{\vee} x=z \cdot u+v\right) \text {, } \\
& u=2^{y} \leftrightarrow \underset{x<u}{\vee}\left(x=y \wedge u=2^{x}\right), \\
& x=y_{1} k\left(\tau_{2}\right) \ldots k\left(\tau_{r}\right)+\cdots+y_{r-1} k\left(\tau_{r}\right)+y_{r} \\
& \leftrightarrow \underset{z_{1}, \ldots, z_{r} \leqq x}{\vee}\left(x=z_{1}+\cdots+z_{r} \wedge \underset{1 \leqq i \leqq r}{\wedge} z_{i}=y_{i} k\left(\tau_{i+1}\right) \ldots k\left(\tau_{r}\right)\right), \\
& z_{i}=y_{i} k\left(\tau_{i+1}\right) \ldots k\left(\tau_{r}\right) \\
& \leftrightarrow\left(y_{i}=0 \wedge z_{i}=0\right) \vee \\
& \vee\left(y_{i} \neq 0 \wedge \underset{x_{i+1}, \ldots, x_{r} \leqq x_{i}}{ }\left({ }_{i+1 \leqq j \leqq r}^{\wedge} x_{j}=k\left(\tau_{j}\right) \wedge z_{i}=y_{i} x_{i+1} \ldots x_{r}\right)\right), \\
& x=k\left(\left(\tau_{1}, \ldots, \tau_{r}\right)\right) \leftrightarrow \bigvee_{y<x}^{\vee}\left(y=k\left(\tau_{1}\right) \ldots k\left(\tau_{r}\right) \wedge x=2^{y}\right) \text {, } \\
& x=0 \leftrightarrow x+x=x \text {. }
\end{aligned}
$$

Mit ihrer Hilfe erhält man aus $\beta(\varphi)$ einen äquivalenten pränexen, abgeschlossenen Ausdruck, dessen Kern nur noch Primformeln der Form $x+y=z, x \cdot y=z$, $2^{x}=y, x=m$ enthält und in dessen Präfix alle Quantoren in der Form $x<y$, $x \leqq y$ oder $x<k(\tau)$ mit in $\varphi$ vorkommenden Typen $\tau$ beschränkt sind. Da alle in $\varphi$ vorkommenden Typen $\tau$ Stufen $\leqq n$ besitzen, gilt mit einem genügend großen $r$ für alle diese Typen $k(\tau)<\vartheta_{n}\left(2^{r}\right)$. Beachtet man noch

$$
\bigwedge_{x<k(\tau)} \beta \leftrightarrow \bigwedge_{x<\vartheta_{n}\left(m^{r}\right)}(x<k(\tau) \rightarrow \beta), \quad x<k(\tau) \leftrightarrow \neg \bigvee_{y \leqq x}^{\bigvee} y=k(\tau)
$$

und die letzten drei Äquivalenzen der obigen Liste, so folgt, daß man $\beta(\varphi)$ äquivalent umformen kann in einen pränexen, abgeschlossenen Ausdruck, dessen Kern wieder aus Primformeln der Form $x+y=z, x \cdot y=z, 2^{x}=y, x=m$ aufgebaut ist und in dessen Präfix jetzt alle Quantoren in der Form $x<y, x \leqq y$ oder $x<\vartheta_{n}\left(m^{r}\right)$ beschränkt sind. Die Primformeln $x=m$ lassen sich eliminieren mit Hilfe der Äquivalenzen

$$
\begin{aligned}
& x=m \leftrightarrow \underset{y<x}{\wedge} y<m \wedge \neg x<m \\
& x<m \leftrightarrow \underset{y<\vartheta_{n}\left(m^{r}\right)}{\vee} y=\vartheta_{n}\left(x^{r}\right) \\
& y=\vartheta_{n}\left(x^{r}\right) \leftrightarrow \underset{z_{0}, \ldots, z_{n-1}<y}{\vee}\left(z_{0}=x^{r} \wedge_{0 \leqq i<n-1}^{\wedge} z_{i+1}=2^{z_{1}} \wedge y=2^{z_{n-1}}\right) \\
& z=x^{r} \leftrightarrow \underset{y_{1}, \ldots, y_{r-1} \leqq z}{\vee}\left(y_{2}=x \cdot x \wedge_{2 \leqq i<r-1}^{\wedge} y_{i+1}=y_{i} \cdot x \wedge z=y_{r-1} \cdot x\right) .
\end{aligned}
$$


Schließlich sind auch noch die in der Form $x<y, x \leqq y$ beschränkten Quantoren eliminierbar. Denn zunächst ist

$$
\wedge_{x<y} \beta \leftrightarrow \bigwedge_{x \leqq y}(\underset{z \leqq y}{\vee}(z+z \neq z \wedge x+z=y) \rightarrow \beta)
$$

Beachtet man jetzt, daß es sich um einen abgeschlossenen Ausdruck handelt, dessen Quantoren, sofern sie nicht durch Variable in der Form $x \leqq y$ beschränkt sind, alle durch $\vartheta_{n}\left(m^{r}\right)$ beschränkt sind, so ergibt sich, daß man alle Teilausdrücke der Form äquivalent ersetzen kann durch $\quad \hat{x \leqq y}_{\leqq} \beta$

$$
\wedge_{x<\vartheta_{n}\left(m^{r}\right)}\left(\underset{z<\vartheta_{n}\left(m^{r}\right)}{\vee} x+z=y \rightarrow \beta\right)
$$

Damit ist gezeigt, daß $\beta(\varphi)$ in einen Ausdruck der gewünschten Form äquivalent umgeformt werden kann, und also der Satz bewiesen.

Satz 3. Zu jedem exponentiell arithmetischen abgeschlossenen Ausdruck $\alpha$ und jedem $n \geqq 1, r \geqq 1$ gibt es einen Ausdruck $\varphi$ der schwachen $n+1$-ten Stufe, so da $\beta$ für alle $m \geqq 1$ gilt: erf $m \varphi$ genau dann, wenn $\alpha$ (im Standardsinn) gültig ist in $\left\{x \mid x<\vartheta_{n}\left(m^{r}\right)\right\}$.

Beweis. Sei $\sigma_{1}=(\iota \ldots \ell)$ mit $r$ Vorkommen von $\iota, \sigma_{i+1}=\left(\sigma_{i}\right) . u, v, w$ seien Variable des Typs $\sigma_{n-1}$, falls $n \geqq 2$, und $r$-Tupel von Variablen des Typs $\iota$, falls $n=1$. Mit großen lateinischen Buchstaben bezeichnen wir Variable der Stufe $n$; insbesondere haben $P, Q, R, P_{1}, \ldots$ den Typ $\sigma_{n}$ und $K$ den Typ $\left(\sigma_{n-1}, \sigma_{n-1}\right)$, falls $n \geqq 2$, und den Typ $(\iota \ldots \ell)$ mit $2 r$ Vorkommen von $\ell$, falls $n=1$. Die Typen der restlichen verwendeten Variablen ergeben sich aus dem Zusammenhang.

Uber einem $m$-elementigen Bereich gibt es $\vartheta_{i}\left(m^{r}\right)$ Objekte des Typs $\sigma_{i}$; dies gilt auch für $i=0$, wenn man unter Objekten des Typs $\sigma_{0} r$-Tupel von Elementen des Bereichs versteht. Ist eine lineare Ordnung $\boldsymbol{K}$ der Objekte des Typs $\sigma_{n-1}$ gegeben, so kann man in Abhängigkeit von $\boldsymbol{K}$ die Objekte des Typs $\sigma_{n}$ eineindeutig auf die 0-1-Folgen der Länge $\vartheta_{n-1}\left(m^{r}\right)$ abbilden: In der $\boldsymbol{P}$ entsprechenden Folge soll die (von hinten) $i$-te Stelle genau dann mit einer 1 besetzt sein, wenn $\boldsymbol{P}$ auf das (bzgl. $\boldsymbol{K}$ ) $i$-te Objekt des Typs $\sigma_{n-1}$ zutrifft. Solche 0-l-Folgen lassen sich wieder eineindeutig auf $\left\{x \mid x<\vartheta_{n}\left(m^{r}\right)\right\}$ abbilden, indem man jede Folge als Dualdarstellung einer Zahl auffaßt. In Abhängigkeit von $\boldsymbol{K}$ hat man also eine eineindeutige Entsprechung zwischen Objekten des Typs $\sigma_{n}$ und Zahlen $<\vartheta_{n}\left(m^{r}\right)$.

Wir definieren jetzt zu jedem e. a. Ausdruck $\beta$ durch Induktion über den Aufbau von $\beta$ einen Ausdruck $\psi(\beta)$ der schwachen $n+1$-ten Stufe. Dabei legen wir eine eineindeutige Zuordnung von Variablen des Typs $\sigma_{n}$ zu Zahlvariablen zugrunde. $\psi(\beta)$ soll folgende Eigenschaften haben: (i) Sind $x_{1}, \ldots, x_{s}$ die freien Variablen von $\beta$, so sollen $K, P_{1}, \ldots, P_{s}$ die freien Variablen von $\psi(\beta)$ sein, wobei $P_{1}, \ldots, P_{s}$ die $x_{1}, \ldots, x_{s}$ zugeordneten Variablen sind. (ii) Interpretiert $\operatorname{man} \beta$ über $\left\{x \mid x<\vartheta_{n}\left(m^{r}\right)\right\}$ und $\psi(\beta)$ über einem $m$-elementigen Bereich, so daß $K$ durch eine lineare Ordnung $\boldsymbol{K}$ und sich entsprechende Variablen in $\beta$ und $\psi(\beta)$ durch bzgl. $\boldsymbol{K}$ sich entsprechende Objekte interpretiert werden, so erhalten $\beta$ und $\psi(\beta)$ denselben Wahrheitswert. 
Fall 1. $x+y=z$. Die $x, y, z$ zugeordneten Variablen vom Typ $\sigma_{n}$ bezeichnen wir (hier und im folgenden) mit $P, Q, R$. Wir verwenden die Abkürzungen $N u v$ für $K u v \wedge \neg \underset{w}{\vee}(K u w \wedge K w v), A u$ für $\neg \neg_{v}^{\bigvee} K v u, E u$ für $\neg \underset{v}{\bigvee} K u v ; \psi(x+y=z)$ sei dann der Ausdruck

$$
\begin{aligned}
P+Q=R: & \equiv \underset{S}{\vee}\left[\wedge ( A u \rightarrow \neg S u ) \wedge \underset { u u ^ { \prime } } { \wedge \wedge } \left(N u u ^ { \prime } \rightarrow \left(S u^{\prime} \leftrightarrow(P u \wedge Q u) \vee(P u \wedge S u) \vee\right.\right.\right. \\
& \wedge \wedge_{u}(R u \leftrightarrow(P u \wedge \neg Q u \wedge \neg S u) \vee(\neg P u \wedge Q u \wedge \neg S u) \vee \\
& \quad \vee(\neg P u \wedge \neg Q u \wedge S u) \vee(P u \wedge Q u \wedge S u)) \wedge \\
& \left.\wedge{ }_{u}(E u \rightarrow(\neg P u \wedge \neg Q u) \vee(\neg P u \wedge \neg S u) \vee(\neg Q u \wedge \neg S u))\right] .
\end{aligned}
$$

Zu zeigen sind (i) und (ii), (i) ist (hier und im folgenden) offensichtlich erfüllt. Zum Beweis von (ii) nehmen wir an, daß $x, y, z$ durch Zahlen $\boldsymbol{x}, \boldsymbol{y}, \boldsymbol{z}<\vartheta_{n}\left(m^{r}\right)$ interpretiert sind. Weiter sei über einem $m$-elementigen Bereich $K$ interpretiert durch eine lineare Ordnung $\boldsymbol{K}$ der Objekte vom Typ $\sigma_{n-1}$, und $P, Q, R$ interpretiert durch die Objekte $\boldsymbol{P}, \boldsymbol{Q}, \boldsymbol{R}$ vom Typ $\sigma_{n}$, die den Zahlen $\boldsymbol{x}, \boldsymbol{y}, \boldsymbol{z}$ entsprechen (bzgl. $\boldsymbol{K}$ ); $\boldsymbol{x}, \boldsymbol{y}, \boldsymbol{z}$ und $\boldsymbol{P}, \boldsymbol{Q}, \boldsymbol{R}$ lassen sich also durch jeweils dieselben 0-1-Folgen der Länge $\vartheta_{n-1}\left(m^{r}\right)$ darstellen. Gilt nun $\boldsymbol{x}+\boldsymbol{y}=\boldsymbol{z}$, so erzeugt das übliche Additionsschema für Zahlen in Dualdarstellung aus den $\boldsymbol{x}, \boldsymbol{y}$ darstellenden 0-1-Folgen die $\boldsymbol{z}$ darstellende 0-1-Folge. Mit Hilfe des Objekts $\boldsymbol{S}$ vom Typ $\sigma_{n}$, das durch die 0-1-Folge des Ubertrags dargestellt wird, ergibt sich die Gültigkeit von $\boldsymbol{P}+\boldsymbol{Q}=\boldsymbol{R}$. Gilt umgekehrt $\boldsymbol{P}+\boldsymbol{Q}=\boldsymbol{R}$, so ergibt die Dualaddition der $\boldsymbol{P}, \boldsymbol{Q}$ darstellenden 0 -1Folgen die $\boldsymbol{R}$ darstellende 0-1-Folge; es gilt also auch $\boldsymbol{x}+\boldsymbol{y}=\boldsymbol{z}$.

Beispiel (Nullen vor der ersten Eins sind weggelassen):

$$
\begin{array}{rr}
\boldsymbol{x}, \boldsymbol{P}: & 1101 \\
\boldsymbol{y}, \boldsymbol{Q}: & 10111 \\
\boldsymbol{S}: & 111110 \\
\boldsymbol{z}, \boldsymbol{R}: & 100100 .
\end{array}
$$

Fall 2. $x \cdot y=z$.

$\psi(x \cdot y=z)$ sei der Ausdruck

$$
\begin{aligned}
& P \cdot Q=R: \equiv \underset{S}{\vee} \underset{T}{\vee}[\underset{w}{\Lambda}(A w \rightarrow \underset{u}{\Lambda} \underset{v}{\Lambda}(S u v w \leftrightarrow P u \wedge Q v)) \wedge \\
& \wedge \bigwedge_{w} \bigwedge_{w^{\prime}}\left(N w w ^ { \prime } \rightarrow \bigwedge _ { u } \bigwedge _ { v } \left(\left[\neg K w v \wedge\left(S u v w \leftrightarrow S u v w^{\prime}\right)\right] \vee\right.\right. \\
& \left.\left.\vee\left[K w v \wedge\left(S u v w \leftrightarrow \underset{u^{\prime}}{\bigvee}\left(N u u^{\prime} \wedge S u^{\prime} v w^{\prime}\right)\right) \wedge\left(A u \rightarrow \neg S u v w^{\prime}\right)\right]\right)\right) \wedge \\
& \wedge \underset{w}{\Lambda}\left(E w \rightarrow \bigwedge_{v}\left(A v \rightarrow \bigwedge_{u}(T u v \leftrightarrow S u v w)\right) \wedge\right. \\
& \left.\wedge \wedge_{v} \underset{v^{\prime}}{\wedge}\left(N v v^{\prime} \rightarrow \lambda u T u v^{\prime}=\lambda u T u v+\lambda u S u v^{\prime} w\right)\right) \wedge \\
& \left.\wedge \wedge_{v}^{\wedge}(E v \rightarrow \underset{u}{\wedge}(R u \leftrightarrow T u v))\right] .
\end{aligned}
$$


Dabei ist $\lambda u T u v^{\prime}=\lambda u T u v+\lambda u S u v^{\prime} w$ der Ausdruck, der aus dem oben angegebenen Ausdruck $P+Q=R$ durch Umbenennen der gebundenen Variablen $S$ und Ersetzen von $P u, Q u, R u$ durch $T u v, S u v^{\prime} w, T u v^{\prime}$ entsteht.

Anstelle eines allgemeinen Beweises für (ii) geben wir ein Beispiel. Das Prädikat $\mathbf{S}$ ist dabei dargestellt durch seine Schnitte bzgl. der letzten Argumentstelle(n). $\boldsymbol{x}, \boldsymbol{P}$ :

$\boldsymbol{y}, \boldsymbol{Q}$ :

10111

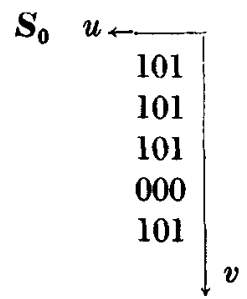

$S_{2} u$

101

1010

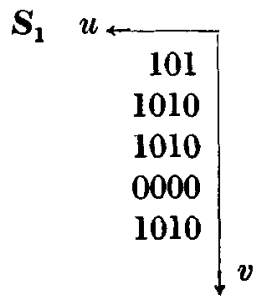

$\boldsymbol{S}_{\theta_{n-1}\left(m^{\prime}\right)} u$

10100

00000

10100

T $u$

\section{$\boldsymbol{z}, \boldsymbol{R}:$}

1110011

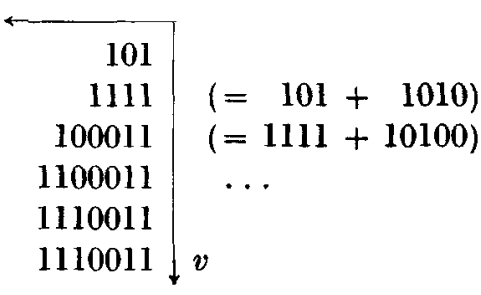

Fall 3. $2^{y}=z$. Wir behandeln allgemeiner den Fall $x^{y}=z$, da dies keine zusätzlichen Schwierigkeiten macht. $\psi\left(2^{y}=z\right)$ läßt sich dann definieren als

$$
\begin{aligned}
& 2^{O}=R: \equiv \underset{P}{\mathrm{~V}}\left(P^{Q}=R \wedge \bigwedge_{u}^{\wedge}(P u \leftrightarrow \underset{v}{\mathrm{~V}}(A v \wedge N v u))\right) \\
& \psi\left(x^{y}=z\right) \text { sei der Ausdruck } \\
& P^{Q}=R: \equiv \underset{S}{\bigvee}\left[\wedge_{v}(E v \rightarrow \underset{u}{\Lambda}(S u v \leftrightarrow A u)) \wedge\right. \\
& \wedge \wedge_{v v^{\prime}}\left(N v v^{\prime} \rightarrow\left[\neg Q v \wedge \lambda u S u v=\lambda u S u v^{\prime} \cdot \lambda u S u v^{\prime}\right] \vee\right. \\
& \left.\vee\left[Q v \wedge \underset{T}{\vee}\left(T=\lambda u S u v^{\prime} \cdot \lambda u S u v^{\prime} \wedge \lambda u S u v=T \cdot P\right)\right]\right) \wedge \\
& \left.\wedge \wedge_{v}(A v \rightarrow \underset{u}{\wedge}(R u \leftrightarrow S u v))\right]
\end{aligned}
$$


Anstelle eines Beweises für (ii) geben wir wieder ein Beispiel:

$$
\begin{aligned}
& \boldsymbol{x}, \boldsymbol{P}: \quad 11 \\
& \boldsymbol{y}, \boldsymbol{Q}: \quad 110
\end{aligned}
$$

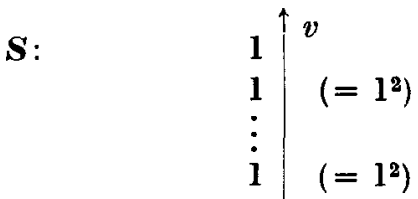

$$
\begin{aligned}
& 11 \quad\left(=1^{2} \cdot 11\right) \\
& 11011 \quad\left(=11^{2} \cdot 11\right) \\
& 1011011001 \quad\left(=11011^{2}\right)
\end{aligned}
$$

Fall 4-6. Wir setzen

$$
\psi(\neg \beta) \equiv \neg \psi(\beta), \quad \psi\left(\beta_{1} \wedge \beta_{2}\right) \equiv \psi\left(\beta_{1}\right) \wedge \psi\left(\beta_{2}\right), \quad \psi(\underset{x}{\wedge} \beta) \equiv \hat{p}_{p} \psi(\beta) .
$$

(ii) ergibt sich unmittelbar aus der Induktionsvoraussetzung.

Ist jetzt $\alpha$ ein e. a. abgeschlossener Ausdruck und definiert man $\varphi: \equiv \bigvee_{K}^{\vee}\left[\Lambda_{u} \neg K u u \wedge \bigwedge_{u} \wedge(K u v \vee K v u \vee u=v) \wedge \bigwedge_{u} \wedge \Lambda_{w}(K u v \wedge K v w \rightarrow K u w) \wedge \psi(\alpha)\right]$, so gilt wegen der Eigenschaften (i), (ii) von $\psi(\alpha)$, daß $\operatorname{erf} f_{m} \varphi$ genau dann, wenn $\alpha$ gültig ist in $\left\{x \mid x<\vartheta_{n}\left(m^{r}\right)\right\}$.

Sei $\mathfrak{M}_{n+1}^{-}:=\{S(\varphi) \mid \varphi$ Ausdruck der schwachen $n+1$-ten Stufe $\}$. Aus den Sätzen 2 und 3 folgt sofort

Korollar 1. $\mathfrak{M}_{n+1}^{-}$ist das System aller Urbilder von Spektren exponentiell arithmetischer Ausdrücke unter Funktionen der Form $\lambda m \vartheta_{n}\left((m+1)^{r}\right)-1, r \geqq 1$.

Beschränkt exponentiell arithmetische (b. e. a.) Ausdrücke seien $x+y=z$, $x \cdot y=z, 2^{x}=y$ (die mit $x, y, z$ bezeichneten Variablen sind nicht notwendig verschieden) und mit $\alpha, \beta$ auch $\neg \alpha,(\alpha \wedge \beta), \underset{x<y}{\wedge} \alpha$. Ein zahlentheoretisches Prädikat $P$ heißt b. e. a., wenn es einen b. e. a. Ausdruck $\alpha\left(x_{1}, \ldots, x_{r}\right)$ gibt, in dem genau die angegebenen Variablen frei vorkommen, so daß $\boldsymbol{P}$ auf $\boldsymbol{x}_{1}, \ldots, \boldsymbol{x}_{r}$ genau dann zutrifft, wenn $\alpha\left(\boldsymbol{x}_{1}, \ldots, \boldsymbol{x}_{r}\right)$ gültig ist.

Korollar 2. $\mathfrak{M}_{n+1}^{-}$ist das System aller Urbilder beschränkt exponentiell arithmetischer Mengen $\left.{ }^{1}\right)$ unter Funktionen der Form $\lambda m \vartheta_{n}\left((m+1)^{r}\right), r \geqq 1$.

Beweis. Daß sich jedes $S \in \mathfrak{M}_{n+1}^{-}$als ein solches Urbild darstellen läßt, ist eine direkte Konsequenz aus Satz 2. Sei umgekehrt $\beta(x)$ ein b. e. a. Ausdruck mit $x$ als einziger freien Variablen. Mit der beim Beweis von Satz 2 angewandten Methode kann man dann einen b. e. a. Ausdruck $\alpha(x)$ konstruieren, in dem $x$ nur in Quantoren-

1) Vgl. Fußnote 2 auf S. 1. 
schranken vorkommt und die einzige freie Variable ist, so daß $\beta\left(\vartheta_{n}\left((m+1)^{r}\right)\right)$ genau dann gültig ist, wenn $\alpha\left(\vartheta_{n}\left((m+1)^{r+1}\right)\right)$ gültig ist. Mit Satz 3 folgt die Behauptung.

Korollar 3, (i) $\operatorname{Pr}^{1}\left(\subseteq_{n}\right) \subseteq \mathfrak{M}_{n+1} \cong \operatorname{Pr}^{1}\left(\subseteq_{n+1}\right), \quad$ (ii) $\mathfrak{M}_{n} \subset \mathfrak{M}_{n+2}$, (iii) $\cup_{n} \mathfrak{M}_{n}=$ $=\operatorname{Pr}^{1}(\mathbb{E})$.

Beweis. Die erste Inklusion von (i) ist Satz 1; die zweite ergibt sich aus $\mathfrak{M}_{n+1} \subseteq$ $\subseteq \mathfrak{M}_{n+2}^{-}$, Korollar 2 und den in $\S 1$ aufgelisteten Eigenschaften der $\mathfrak{S}_{n}$. (ii) und (iii) folgen unter Verwendung von $\S 1$ aus (i).

\section{Literatur}

[1] Asser, G., Das Repräsentantenproblem im Prädikatenkalkül der ersten Stufe mit Identität. Diese Zeitschr. 1 (1955), 252-263.

[2] Grzegorczy K, A., Some classes of recursive functions. Rozprawy Matematyczne IV, Warzsaw 1953.

[3] KLEENE, S. C., Introduction to metamathematics. Amsterdam 1964.

[4] Mostowskr, A., Concerning a problem of H. Scholz. Diese Zeitschr. 2 (1956), 210-214.

[5] Röddine, D., Klassen rekursiver Funktionen. In: Proceedings of the Summer School in Logic, Leeds, 1967. Berlin-Heidelberg-New York 1968, S. 159-222.

[6] Scholz, H., Ein ungelöstes Problem in der symbolischen Logik. J. Symb. Logic 17 (1952), 160.

[7] Shepherdson, J. C., and H. E. Sturgis, Computability of recursive functions. J. Ass. Computing Mach. 10 (1963), 217-255.

[8] Suullyan, R. M., Theory of formal systems. Princeton 1961.

(Eingegangen am 26. Februar 1971) 